\title{
Alexithymia predicts loss chasing for people at risk for problem gambling
}

\author{
PETER A. BIBBY* and KATHERINE E. ROSS \\ School of Psychology, University of Nottingham, Nottingham, United Kingdom \\ (Received: February 6, 2017; revised manuscript received: July 31, 2017; second revised manuscript received: September 19, 2017; \\ accepted: October 22, 2017)

\begin{abstract}
Background and aims: The aim of this research was to investigate the relationship between alexithymia and losschasing behavior in people at risk and not at risk for problem gambling. Methods: An opportunity sample of 58 (50 males and 8 females) participants completed the Problem Gambling Severity Index and the Toronto Alexithymia Scale (TAS-20). They then completed the Cambridge Gambling Task from which a measure of loss-chasing behavior was derived. Results: Alexithymia and problem gambling risk were significantly positively correlated. Subgroups of non-alexithymic and at or near caseness for alexithymia by low risk and at risk for problem gambling were identified. The results show a clear difference for loss-chasing behavior for the two alexithymia conditions, but there was no evidence that low and at-risk problem gamblers were more likely to loss chase. The emotion-processing components of the TAS-20 were shown to correlate with loss chasing. Discussion and conclusion: These findings suggest that loss-chasing behavior may be particularly prevalent in a subgroup of problem gamblers those who are high in alexithymia.
\end{abstract}

Keywords: alexithymia, problem gambling, loss chasing

\section{INTRODUCTION}

Alexithymia (Sifneos, 1973) is a stable personality trait characterized by difficulties in processing emotional information and a tendency to use an external cognitive style (Parker, Taylor, \& Bagby, 2001; Taylor, Bagby, \& Parker, 1997). Alexithymia and problem gambling have been shown to be associated in a number of studies. For example, Lumley and Roby (1995) found in a sample of adolescent problem gamblers, $31.4 \%$ were alexithymic compared with only $11 \%$ in healthy controls. Parker, Wood, Bond, and Shaughnessy (2005) found that $22 \%$ of a sample of pathological gamblers were alexithymic compared with $11 \%$ on non-problem gamblers. Bonnaire, Bungener, and Varescon (2009) found a rate of $44 \%$ of problem gamblers being rated as high in alexithymia. Toneatto, Lecce, and Bagby (2009) found that as problem gambling risk and intensity increased so did the level of alexithymia. A significantly higher proportion of pathological gamblers $(76 \%)$ were identified as alexithymic compared with $55 \%$ of problem gamblers. Both groups were significantly more likely to be alexithymic than the non-problem gamblers (29\%). Bonnaire, Bungener, and Varescon (2013) found that pathological gamblers who gambled at race tracks or played slot machines had higher overall alexithymia scores than non-pathological gamblers. Bonnaire et al. (2017) found an association between alexithymia and pathological gamblers for strategic gamblers, i.e., people who engaged in: gambling at race tracks, offcourse betting, sports betting, or card games. Not only is alexithymia associated with problem gambling, the degree of alexithymia and the severity of the problem gambling (Di Trani, Renzi, Vari, Zavattini, \& Solano, 2017; Maniaci et al., 2015; Mitrovic \& Brown, 2009) are also associated with it. In other words, those higher in alexithymia show more of the indicators of problem gambling than a simple association might indicate.

Loss chasing, continuing to bet in an attempt to recover from an earlier loss, is a common feature of gambling. Loss chasing is not always problematic, but it can be a critical step toward harmful gambling behavior (Dickerson, Hinchy, \& Fabre, 1987). The fifth edition of the Diagnostic and Statistical Manual of Mental Disorders (American Psychiatric Association, 2013) recognizes the dangers of chasing and includes it as part of the diagnostic criteria for problem gambling. Gambling screens also include loss-chasing items to identify those at risk of problems; e.g., the Problem Gambling Severity Index (PGSI; Ferris \& Wynne, 2001) and the South Oaks Gambling Screen (Lesieur \& Blume, 1987). In a study conducted at gambling venues, an analysis of retrospective questionnaires shows that chasing predicts extreme gambling behavior and poor control scores (O’Connor \& Dickerson, 2003).

These gambling screens specifically refer to loss chasing as occurring between-gambling sessions where there is a return on a separate occasion to recoup previous losses. However, loss chasing can also occur within a gambling

\footnotetext{
* Corresponding author: Dr. Peter A. Bibby; School of Psychology, University of Nottingham, University Park, Nottingham NG7 2RD, United Kingdom; Phone: +44 115951 5329; Fax: +44 115 951 5324; E-mail: peter.bibby@nottingham.ac.uk
} 
session (Breen \& Zuckerman, 1999). Indeed, given the prevalence of online and electronic gambling portals that encourage continuous play within-session loss chasing may be of increasing importance. In a recent study, using the Iowa Gambling Task (IGT; Bechara, Damasio, Damasio, \& Anderson, 1994), a measure of risky decision-making, Linnet, Rojskjaer, Nygaard, and Maher (2006) demonstrated that, although within-session loss chasing was common across all participants, it was particularly prevalent in problem gamblers.

Ferguson et al. (2009) suggested that alexithymia was associated with a reduced sensitivity to losses in the IGT. Using both riskless and risky loss-aversion tasks, Bibby and Ferguson (2011) found that alexithymia was associated with a reduction in loss aversion. Bibby (2016) argued that loss chasing when gambling might reflect this reduced loss aversion in alexithymics. He demonstrated that within-session loss chasing was associated with alexithymia in a sample of university students. Bibby compared the proportion of the available betting stake gambled after a win or a loss. He found that participants high in alexithymia bet more after a loss than a win when the proportion bet was both low and high, but nonalexithymic participants tended only to chase losses after a high proportion of the available stake had been bet. Consistent with the idea that problem gambling in alexithymics might be associated with poor emotional processing, it was the emotional-processing components of alexithymia that were correlated with the loss-chasing behavior. The difficulty identify feelings (DIF) and difficulty describing feelings (DDF) subdimensions of the alexithymia construct (Bagby, Parker, \& Taylor, 1994) were found to correlate with loss chasing, but the externally oriented thinking (EOT) component did not.

This paper seeks to extend the work of Bibby (2016) to a sample of gamblers. To this purpose, participants were recruited from betting shops in London. It was hypothesized that the participants' alexithymia scores would be positively correlated with their problem gambling risk scores. A second hypothesis was that participants' loss-chasing behavior would be associated with alexithymia due to alexithymia being related to reduced loss aversion. Essentially, this would be a replication of the effect observed by Bibby (2016) but for a sample of adults who gamble.

Given that loss chasing is identified as a clinical criterion for problem gambling, it can also be hypothesized that those at greater risk for problem gambling would loss chase more than those at low risk for loss chasing. However, while a psychological mechanism has been described for the association between loss chasing and alexithymia, no such explanation exists for the relationship between loss chasing and problem gambling. It could be the case given the associations between loss chasing and alexithymia and between problem gambling and alexithymia that it is actually alexithymia that predisposes people to loss chasing. In this case, it is possible to hypothesize that it is not being at risk for problem gambling that is associated with loss chasing. In such case, an independent effect of problem gambling risk might not be observed.

Two hypotheses for the interaction between alexithymia and loss chasing are possible. First, there is no interaction between alexithymia and problem gambling risk. Whatever the underlying psychological mechanisms that lead to loss chasing, alexithymia and problem gambling risk may be independent. Second, the combination of high alexithymia and greater risk of problem gambling raises the likelihood of loss chasing even further. In this case, the effect of alexithymia on loss chasing is moderated by problem gambling risk.

\section{METHODS}

\section{Participants}

An opportunity sample of 58 participants was recruited across six betting shops in London (50 males and 8 females). The average age was 48.1 years, $S D=13.46$. Participation was not incentivized due to compliance with UK gambling licensing regulations. This represents a response rate of approximately $39 \%(58 / 150)$ of those who were asked to participate. A cut-off point of Toronto Alexithymia Scale (TAS-20) < 52 was used (Bagby \& Taylor, 1997, p. 62) with those scoring less identified as non-alexithymic and those above identified as at or near caseness for alexithymia. There were 32 participants who screened as non-alexithymic and 26 who screened as at or near caseness for alexithymia. A higher cut-off point $(\geq 61)$ for defining participants as clearly alexithymic (Bagby \& Taylor, 1997, p. 62) meant that there were too few participants $(N=4)$ in the alexithymic lower risk for problem gambling group for satisfactory statistical analysis of the data. However, using this higher cut-off, the $31 \%$ prevalence of alexithymia in this sample is more than expected for non-clinical samples. Using the same cut-off point $(\geq 61)$ as Parker, Keefer, Taylor, and Bagby (2008), the observed incidence of $31 \%$ in this sample is significantly different from the prevalence of $10 \%$ they observed in a community sample $(N=1,933 ; z=5.12$, $p<.001)$ and $11 \%$ in an undergraduate sample $(n=$ $1,948 ; z=4.69, p<.001)$. The PGSI was used to identify 28 participants at lower risk of problem gambling and 30 participants at higher risk. The lower risk group was formed by collapsing the no risk $(n=12)$, lower risk $(n=4)$, and medium risk $(n=12)$ classifications from the PGSI into a single group. The remaining 30 participants were classified as higher risk $(n=30)$ as per the PGSI classification.

Analyses of variance (ANOVAs) demonstrated that for the TAS-20, there was a significant main effect for alexithymia $\left(F_{1,54}=127.71, \mathrm{MSE}=47.89, p<.001, \eta_{p}^{2}=0.70\right)$ but not for problem gambling risk $\left(F_{1,54}=3.32, \mathrm{MSE}=\right.$ $\left.47.89, p=.08, \eta_{p}^{2}=0.06\right)$. The interaction was not statistically significant $\left(F_{1,54}=0.40, \mathrm{MSE}=47.89, p=.53, \eta_{p}^{2}=\right.$ $0.01)$. For the PGSI, the effect for alexithymia was not significant $\left(F_{1,54}=1.15, \mathrm{MSE}=18.17, p=.29, \eta_{p}^{2}=0.02\right)$, but the main effect for problem gambling risk was $\left(F_{1,54}=\right.$ $\left.117.53, \mathrm{MSE}=18.17, p<.001, \eta_{p}^{2}=0.69\right)$. The interaction was not statistically significant $\left(F_{1,54}=.66, \mathrm{MSE}=18.17\right.$, $\left.p=.42, \eta_{p}^{2}=0.01\right)$. The means and standard deviations are shown in Table 1 .

To address the issue of a greater number of male participants, two $\chi^{2}$ tests were performed. The distribution of sex by alexithymia $\left(\chi^{2}=0.201, p=.654\right)$ and sex and problem 
Table 1. Means (and standard errors) of the TAS-20 and PGSI scores for the alexithymia by PGSI groups

\begin{tabular}{llcc}
\hline Alexithymia & $\begin{array}{c}\text { Problem } \\
\text { gambling risk }\end{array}$ & $\begin{array}{c}\text { TAS-20 } \\
\text { scores }\end{array}$ & $\begin{array}{c}\text { PGSI } \\
\text { scores }\end{array}$ \\
\hline Non- & Lower $(N=20)$ & $39.45(1.73)$ & $1.50(.44)$ \\
alexithymic & Higher $(N=12)$ & $41.75(1.26)$ & $15.42(1.74)$ \\
At or near & Lower $(N=8)$ & $60.13(1.42)$ & $3.75(.84)$ \\
caseness & Higher $(N=18)$ & $64.89(2.12)$ & $15.72(1.24)$ \\
\hline
\end{tabular}

Note. TAS-20: Toronto Alexithymia Scale; PGSI: Problem Gambling Severity Index.

gambling risk $\left(\chi^{2}=2.654, p=.103\right)$ were not significant. This suggests that sex is not a confounding variable.

\section{Measures}

Alexithymia was assessed using the 20-item TAS-20 (Bagby et al., 1994). The TAS-20 is a self-report measure of alexithymia that includes 20 items. Items are grouped according to three factors; DIF, DDF, and EOT, which are central to the alexithymia personality trait. Items for each factor include "I am often confused about what emotion I am feeling," "It is difficult for me to find the right words for my feelings," and "I prefer to analyze problems rather than just describe them." Responses are captured using a 1- to 5-point Likert-type scale where $1=$ strongly agree and $5=$ strongly disagree with the statement. Some of the items are reverse-scored and the overall total score is calculated with a higher score indicating higher alexithymia. The TAS-20 showed good internal reliability $(\alpha=0.84)$.

Risk of problem gambling was assessed using the PGSI (Ferris \& Wynne, 2001). The PGSI is an abbreviated version of the Canadian Problem Gambling Index (CPGI) that was developed to measure the prevalence of gambling and problem gambling in the general population. The PGSI is a nine-item self-report questionnaire condensing the original 31 items of the original CPGI (Ferris \& Wynne, 2001).

The PGSI asks the participant to consider their gambling behavior over the past 12 months and evaluate it using a 0 - to 3-point Likert-type scale to respond to the item questions where $0=$ never, $1=$ sometimes, $2=$ most of the time, and $3=$ almost always. The items measure gambling involvement, problem gambling behavior, and adverse consequences and include "How often have you bet more than you could really afford to lose?," "How often have you felt guilty about the way you gamble or what happens when you gamble?," and "Has your gambling caused any financial problems for you or your household?" The score for each item is summed together to give a score ranging from 0 to 27 and the classifications applied are 8 or above $=$ high risk, $3-7=$ moderate risk, $1-2=$ low risk, and $0=$ no risk of problem gambling. The PGSI showed good internal reliability $(\alpha=0.93)$.

An adapted version of the computer-based Cambridge Gambling Task (CGT; Rogers et al., 1999) was used to examine loss-chasing behavior. In the task, 10 boxes were displayed at the top of the screen, a number of which are red and a number of which are blue. On each trial, the number of red or blue boxes was randomly varied between 1 and 9 , so that there was an equal likelihood of each of the nine outcomes.

On-screen instructions told the participant that the computer had randomly hidden a yellow token inside one of the boxes. The placement of the yellow token was randomly varied on each trial between boxes 1 and 10 with an equal likelihood of each box. The participant was asked which color box the yellow token was hidden inside and to indicate their decision by clicking on the "RED" or "BLUE" buttons shown at the bottom of the screen.

To the left of the screen, the points available to bet were shown. The points were incremented with any wins and decremented by losses. If the stake fell below 200 points, it was automatically increased back to 200 points and participants were informed if this occurred by an on-screen message.

The bet to be made was shown in a window at the right of the screen. The value shown in the window decreased in $10 \%$ steps at 1 -s intervals from $90 \%$ of the available stake through $10 \%$ of the available stake. If a participant had not bet after the $1-\mathrm{s}$ interval at the $10 \%$ step, the computer returned to $90 \%$ and began cycling down again. A randomly generated amount of positive/negative error (up to $10 \%$ of the $10 \%$ step) was added to the amount shown to mask the $10 \%$ adjustments.

Participants were required to click a "BET NOW" button when the desired proportion of the stake was shown. Immediately following the selection, the position of the yellow token was displayed in one of the boxes at the top of the screen. If the participant selected the correct color, e.g., if they selected RED and the yellow token was in a red box, then the available stake was increased by the amount bet. If the selection was incorrect, then the available stake was decreased by the amount bet.

The example in Figure 1a shows the screen for a participant who selected "BLUE" and bet 105 out of 200 points. Figure $1 \mathrm{~b}$ shows that the yellow token was in a blue box and since the correct response was given, 105 points were award. The new score is shown in the window at the bottom left of the screen.

\section{Procedure}

Participants first read a description of the planned research and were asked for their consent to participate. Once their informed consent was given, they completed the TAS-20 and PGSI. Next, they completed the adapted CGT [In the original CGT, the proportion available to bet incremental increased from zero in this version, it incrementally decreased from $100 \%$. This adaptation was used since Bibby (2016) found that it increased the likelihood of withingambling-session loss chasing.]. There were 10 practice trials where the betting component was removed after which the task started including betting which lasted for 100 trials. Once the trials were completed, the participant was debriefed and thanked for participating.

Participants completed the research procedure while seated at a table in the bookmaker's premises in which they were recruited. 
(a)

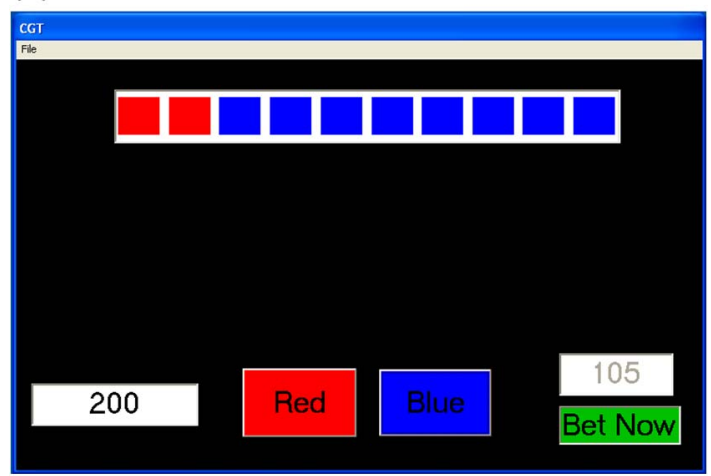

(b)

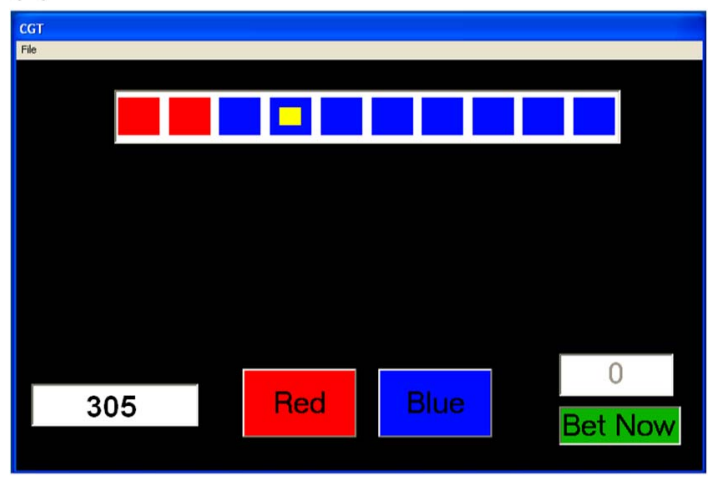

Figure 1. Screen from the Cambridge Gambling Task immediately (a) before a bet is made and (b) after a bet is made

\section{Ethics}

The study procedures were carried out in accordance with the Declaration of Helsinki. The School of Psychology, University of Nottingham ethics committee approved the study. All subjects were informed about the study and all provided informed consent.

\section{RESULTS}

The results of the study are arranged in the following manner. First, the association between alexithymia and risk of problem gambling was examined. Second, a mixed ANOVA was conducted to establish that participants were behaving rationally with respect to the odds of winning. Third, a further mixed ANOVA was conducted to examine loss-chasing behavior. Finally, the correlations between loss chasing, both within- and between-gambling sessions, were examined.

\section{Are alexithymia and problem gambling risk related?}

To test whether there was an association between alexithymia and problem gambling risk, a $\chi^{2}$ test was conducted. The result showed that there was indeed a relationship $\left(\chi^{2}=5.78, p=.02\right.$; see Table 2$)$. Of those participants in the higher risk for problem gambling group, $60 \%$ were at or near caseness for alexithymia, but only $29 \%$ were at or near caseness in the low risk for problem gambling group. The correlation between the TAS-20 score and the PGSI score
Table 2. Number of participants (and percentage) allocated to the alexithymia and problem gambling risk groups

\begin{tabular}{lrc}
\hline & Low risk & At risk \\
\hline Non-alexithymic & $20(34.5 \%)$ & $12(20.7 \%)$ \\
At or near caseness & $8(13.8 \%)$ & $18(31.0 \%)$ \\
\hline
\end{tabular}

was positive and significant $(r=.46, p<.001)$. The higher the level of alexithymia, the participants showed the more likely they are to be at risk of problem gambling.

\section{Do people behave rationally with respect to the odds of winning in the CGT?}

Two participants failed to complete the gambling task and are therefore not included in the following analyses. To examine whether the participants behaved rationally with respect to the CGT, a 2 (alexithymia: non-alexithymic and at or near caseness) $\times 2$ (problem gambling risk: low risk and at risk) $\times 5$ (probability of winning: $p=.5, .6, .7, .8$, and .9) (Throughout the paper, $F$ statistics including this variable were Greenhouse and Geisser corrected to take account of the failure to meet the sphericity assumption) mixed ANOVA was conducted on the proportion of the available stake that was bet. The means and standard errors (SES) can be found in Table 3 .

There was a significant main effect of alexithymia $\left(F_{1}, \quad 52=7.93, \quad \mathrm{MSE}=0.085, p=.01, \eta_{p}^{2}=0.13\right)$. Nonalexithymic participants bet a smaller proportion of the available stake (mean $=0.66, S E=0.02$ ) than those at or near caseness (mean $=0.76, S E=0.03$ ). The main effect of probability of winning was also significant $\left(F_{2.27}, 118.21=\right.$ 15.58, MSE $=0.012, p<.001, \eta_{p}^{2}=0.23$; see Table 3). As the likelihood of winning increased the mean proportion bet also increased. This suggests that participants did indeed understand the gambling task and responded appropriately. The main effect of problem gambling risk was not statistically significant $\left(F_{1,52}=0.24, \mathrm{MSE}=0.085, p=.63, \eta_{p}^{2}=0.01\right)$.

The two-way interaction between alexithymia and problem gambling risk was not significant $\left(F_{1,52}=2.35, \mathrm{MSE}=\right.$ $\left.0.085, p=.14, \eta_{p}^{2}=0.04\right)$. The two-way interaction between alexithymia and probability of winning was also not significant $\left(F_{2.27,118.21}=1.95, \mathrm{MSE}=0.012, p=.11, \eta_{p}^{2}=0.04\right)$. The interaction between problem gambling risk and probability of winning was significant $\left(F_{2.27,118.21}=4.72\right.$, MSE $=$ $0.012, p=.001, \eta_{p}^{2}=0.08$; see Figure 2). The linear trend contrast analysis indicated that the slopes of the two groups for the mean proportion bet were significantly different $\left(F_{1,52}=7.063, \mathrm{MSE}=0.016, p=.01, \eta_{p}^{2}=0.12\right)$. While both problem gambling risk groups bet an increasing mean proportion as the probability of winning increased the rate of change was greater for the at-risk compared with the lowrisk group.

\section{Is loss chasing related to alexithymia, risk of problem gambling, or both?}

To assess whether loss chasing behavior occurred a 2 (alexithymia: non-alexithymic and at or near caseness) $\times$ 
Table 3. Means (and standard errors) of the proportion bet by alexithymia and problem gambling risk by probability of winning on the current trial

\begin{tabular}{llccccc}
\hline & & \multicolumn{3}{c}{ Probability of winning } \\
\cline { 3 - 6 } Alexithymia & Problem gambling risk & $p=.5$ & $p=.6$ & $p=.7$ & $p=.8$ & $p=.9$ \\
\hline \multirow{2}{*}{ Non-alexithymic } & Low $(N=19)$ & $.58(.04)$ & $.60(.03)$ & $.62(.03)$ & $.62(.03)$ & $.68(.03)$ \\
& At risk $(N=12)$ & $.56(.05)$ & $.65(.04)$ & $.69(.04)$ & $.77(.04)$ & $.80(.04)$ \\
At or near caseness & Low $(N=8)$ & $.75(.06)$ & $.78(.05)$ & $.79(.05)$ & $.79(.05)$ & $.79(.05)$ \\
& At risk $(N=17)$ & $.67(.04)$ & $.73(.04)$ & $.75(.04)$ & $.78(.04)$ & $.79(.03)$ \\
& Overall $(N=56)$ & $.64(.03)$ & $.69(.02)$ & $.71(.02)$ & $.74(.02)$ & $.76(.02)$ \\
\hline
\end{tabular}

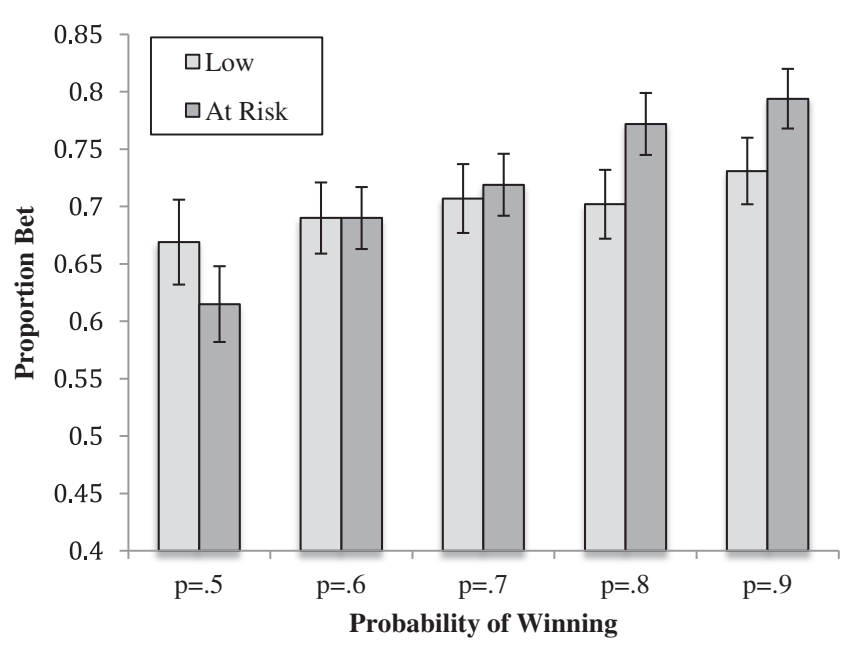

Figure 2. The means (and standard errors) of the proportion bet for the problem gambling low-risk- and at-risk groups

2 (problem gambling risk: low risk and at risk) $\times 2$ (previous trial: loss and win) $\times 5$ (probability of winning: $p=.5, .6, .7$, .8 , and .9) mixed ANOVA was conducted on the proportion of the stake that was bet. The means and SEs are found in Table 4 . The previous trial variable examined the proportion bet after the previous trial was won or lost.

There were significant main effects of alexithymia $\left(F_{1,52}=7.052, \mathrm{MSE}=0.157, p=.01, \eta_{p}^{2}=0.12\right)$, previous trial $\left(F_{1,52}=6.76, \mathrm{MSE}=0.014, p=.01, \eta_{p}^{2}=0.12\right)$, and probability of winning $\left(F_{2.34}, 121.86=11.96, \mathrm{MSE}=0.014\right.$, $\left.p<.001, \eta_{p}^{2}=0.19\right)$. Overall, non-alexithymic participants bet a smaller mean proportion of the available stake (mean $=$ $0.66, S E=0.02)$ than those at or near caseness (mean $=0.75$, $S E=0.03)$. Participants bet more after a loss (mean $=0.72$, $S E=0.02)$ than after a win (mean $=0.69, S E=0.02)$. Furthermore, as the probability of winning increased the mean proportion, bet also increased (Table 4). The main effect of problem gambling risk was not significant $\left(F_{1,52}=0.489\right.$, $\left.\mathrm{MSE}=0.157, p=.49, \eta_{p}^{2}=0.01\right)$.

The two-way interaction between alexithymia and previous trial was significant $\left(F_{1,52}=7.121, \mathrm{MSE}=\right.$ $0.014, p=.01, \eta_{p}^{2}=0.12$; see Figure 3 ) as was the interaction between problem gambling risk and the probability of winning $\left(F_{2.34}, 121.86=3.316, \mathrm{MSE}=0.014, p=.01, \eta_{p}^{2}=\right.$ 0.06). Simple effect analysis indicated that for nonalexithymic participants, the proportion bet was not significantly different after a win or loss on the previous trial $(p=.96)$. However, for those at or near caseness, there was a significant difference $(p=.001)$ with a greater proportion bet after a loss. The alexithymia groups were significantly different after a win $(p=.05)$ and after a loss $(p=.004)$. In both cases, those at or near caseness bet more. For the interaction between problem gambling risk and the probability of winning the interaction linear contrast was significant $\left(F_{1,52}=5.126, \mathrm{MSE}=0.033, p=.03, \eta_{p}^{2}=0.10\right)$.

The two-way interactions between alexithymia and problem gambling risk $\left(F_{1,52}=2.624, \mathrm{MSE}=0.157, p=.11\right.$, $\left.\eta_{p}^{2}=0.05\right)$, alexithymia and probability of winning $\left(F_{2.34,} 121.86=1.527, \mathrm{MSE}=0.024, p=.20, \eta_{p}^{2}=0.03\right)$, problem gambling risk and previous trial $\left(F_{1,52}=0.216\right.$, $\left.\mathrm{MSE}=0.014, p=.64, \eta_{p}^{2}<0.01\right)$, and between previous trial and probability of winning $\left(F_{3.691}, 191.952=1.897\right.$, $\left.\mathrm{MSE}=1.22, p=.11, \eta_{p}^{2}=0.04\right)$ were not statistically significant. The three-way interactions between alexithymia, problem gambling risk, and previous trial $\left(F_{1,52}=1.215\right.$, $\left.\mathrm{MSE}=0.014, p=.28, \eta_{p}^{2}=0.02\right)$, alexithymia, problem gambling risk, and probability of winning $\left(F_{2.34}, 121.862=\right.$ $\left.0.956, \mathrm{MSE}=0.014, p=.432, \eta_{p}^{2}=0.02\right)$, alexithymia, previous trial, and probability of winning $\left(F_{3.691,191.953}=1.534\right.$, $\left.\mathrm{MSE}=1.22, p=.19, \eta_{p}^{2}=0.03\right)$, and problem gambling risk, previous trial, and probability of winning $\left(F_{3.691,191.952}=\right.$ $\left.0.304, \mathrm{MSE}=1.22, p=.88, \eta_{p}^{2}<0.01\right)$ were not statistically significant. Finally, the four-way interaction was not significant $\left(F_{3.691,191.952}=0.527, \mathrm{MSE}=1.22, p=.72, \eta_{p}^{2}=0.01\right)$.

How are within- and between-gambling-session loss chasing related to alexithymia and the risk of problem gambling?

An overall loss-chasing score was constructed by averaging the proportion bet after a loss and subtracting the average of the proportion bet after a win. A simultaneous linear regression was conducted using the TAS-20 score and the PGSI score as predictor variables and the loss-chasing score as the criterion variable. Overall, the model was statistically significant $\left(F_{2}, \quad 53=4.740, \quad \mathrm{MSE}=0.006, \quad p=.01\right.$, adj $\left.R^{2}=.12\right)$. The PGSI was not a statistically significant predictor $(\beta=-0.194, p=.16)$ but the TAS-20 was $(\beta=$ $0.416, p=.003)$. The higher the TAS-20, indicating a greater degree of alexithymia, the greater the loss-chasing score, indicating a bigger difference between the proportion bet after a loss than a win for those higher in alexithymia.

The PGSI includes a between-session loss-chasing item: "When you gambled, did you go back another day to try to win back the money you lost?" This allows the examination of the relationship between- and within-session loss chasing and both kinds of loss chasing and their relationship to 
Table 4. Means (and standard errors) of the proportion bet for alexithymia and impulsivity by outcome of the previous trial and probability of winning on the current trial

\begin{tabular}{|c|c|c|c|c|c|c|c|}
\hline \multirow[b]{2}{*}{ Alexithymia } & \multirow[b]{2}{*}{ Problem gambling risk } & \multirow[b]{2}{*}{ Previous trial } & \multicolumn{5}{|c|}{ Probability of winning } \\
\hline & & & $p=.5$ & $p=.6$ & $p=.7$ & $p=.8$ & $p=.9$ \\
\hline \multirow[t]{4}{*}{ Non-alexithymic } & \multirow[t]{2}{*}{ Low } & Won & $.61(.04)$ & $.60(.04)$ & $.61(.03)$ & $.57(.04)$ & $.66(.03)$ \\
\hline & & Lost & $.58(.05)$ & $.60(.04)$ & $.63(.04)$ & $.64(.04)$ & $.69(.04)$ \\
\hline & \multirow[t]{2}{*}{ At risk } & Won & $.63(.05)$ & $.67(.05)$ & $.68(.04)$ & $.77(.05)$ & $.79(.03)$ \\
\hline & & Lost & $.55(.06)$ & $.65(.05)$ & $.69(.05)$ & $.77(.04)$ & $.80(.05)$ \\
\hline \multirow[t]{5}{*}{ At or near caseness } & \multirow[t]{2}{*}{ Low } & Won & $.70(.06)$ & $.77(.06)$ & $.74(.05)$ & $.78(.05)$ & $.74(.04)$ \\
\hline & & Lost & $.77(.07)$ & $.79(.06)$ & $.81(.06)$ & $.80(.05)$ & $.81(.06)$ \\
\hline & \multirow[t]{3}{*}{ At risk } & Won & $.66(.04)$ & $.69(.04)$ & $.70(.04)$ & $.74(.04)$ & $.75(.03)$ \\
\hline & & Lost & $.71(.05)$ & $.74(.04)$ & $.77(.04)$ & $.80(.04)$ & $.83(.04)$ \\
\hline & & Overall & $.65(.02)$ & $.69(.02)$ & $.71(.02)$ & $.73(.02)$ & $.76(.02)$ \\
\hline
\end{tabular}

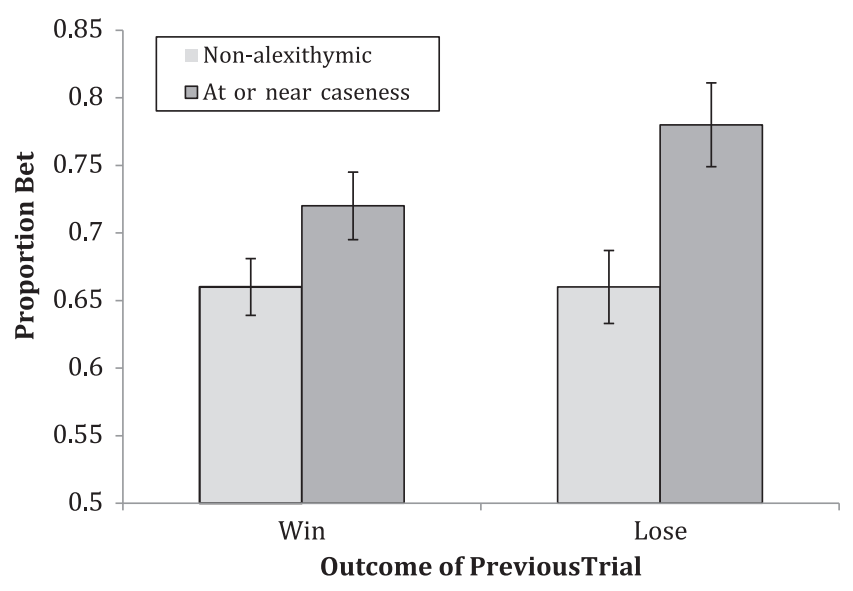

Figure 3. The proportion bet (means and standard errors) for the alexithymia groups having won or lost on the previous trial

alexithymia using the overall TAS-20 score and the TAS-20 subscale scores (Table 5). Given that the subscales of the TAS-20 are based on both separate feelings and cognitive components examining the correlations will further elucidate whether loss chasing is an emotional or cognitive response or a mixture of both. The correlation between the two types of loss chasing was not statistically significant $(r=.115, p=.40)$. However, both between- and withinsession loss chasing were positively correlated with

Table 5. The correlation between the overall and subscales score of the TAS-20 and the between- and within-session loss-chasing measures

\begin{tabular}{lcc}
\hline & $\begin{array}{c}\text { Between-session } \\
\text { loss chasing }\end{array}$ & $\begin{array}{c}\text { Within-session } \\
\text { loss chasing }\end{array}$ \\
\hline TAS-20 overall & $0.317^{*}$ & $0.345^{*}$ \\
$\begin{array}{c}\text { Difficulty describing } \\
\text { feelings }\end{array}$ & 0.213 & $0.418^{* *}$ \\
$\begin{array}{c}\text { Difficulty identifying } \\
\text { feelings }\end{array}$ & $0.309^{*}$ & $0.331^{*}$ \\
$\begin{array}{c}\text { Externally oriented } \\
\text { thinking }\end{array}$ & $0.261^{*}$ & 0.115 \\
\hline
\end{tabular}

Note. TAS-20: Toronto Alexithymia Scale.

${ }^{*} p \leq .05 . * * p \leq .001$. alexithymia. For the between-session loss chasing, the DIF and the EOT alexithymia subscales were positively correlated with loss chasing, but the DDF correlation with between-session loss chasing was not significant. A different pattern of correlations emerged for within-session loss chasing. In this case, both DDF and DIF subscales were positively correlated with loss chasing, but the EOT subscale was not significantly correlated.

\section{DISCUSSION}

In this sample of adult gamblers, the participants who were at risk of problem gambling were twice as likely to be at or near caseness for alexithymia as those at low risk for problem gambling. Furthermore, the correlation between alexithymia (TAS-20) and (PGSI) problem gambling scores was significant and positive with alexithymia accounting for approximately $21 \%$ of the variability in the problem gambling scores. This replicates previous findings of an association between alexithymia and problem gambling (Bonnaire et al., 2009, 2013; Lumley \& Roby, 1995; Parker et al., 2005; Toneatto et al., 2009).

Overall, participants were able to make rational decisions with respect to the gambling task (CGT). As the likelihood of winning increased, then the proportion of the available stake that was gambled also increasing. However, in comparison with Bibby's (2016) university students, the adult gamblers in this study bet proportionally more overall $\left(64.4 \%\right.$ vs. $\left.75.3 \% ; t_{55}=5.77, p<.001\right)$. The greater the amount bet, the larger the potential loss. Bibby (2016) argued that this leads to a stronger cognitive need to loss chase. However, overall, while the loss-chasing effect found in the current research (i.e., the difference between the proportion between after a loss and win, respectively) was statistically significant (3\%), it was less than in the Bibby's (2016) paper (9\%). This reflects an artifactual ceiling effect. There is a maximum of $90 \%$ that can be bet in this task, so the bigger the initial bet, the closer to that maximum, thus reducing the size of the potential loss-chasing effect.

Overall, there was evidence that alexithymia was related to loss chasing. Non-alexithymic participants did not show a statistically different difference between the proportion bet after a loss and the proportion bet after a win (mean 
difference $=0.1 \%$, whereas those at or near caseness demonstrated a difference (5.6\%). However, risk of problem gambling was not significantly associated with loss chasing. Thus, the evidence seems to suggest that loss chasing is associated with alexithymia and not specifically with problem gambling. Given that the preponderance of problem gamblers who tend to be highly alexithymic is much greater than in the general population, then it is not surprising that loss chasing is identified as associated with problem gambling. However, we suggest that this is not an issue of problem gambling per se but rather a problem associated with alexithymia.

The finding that it is alexithymia that matters when loss chasing is supported by a similar result in a recent study that also recruited participants from betting shops (Aïte et al., 2014). In the study, where participants were sampled from sportsbooks betting shops in Paris, alexithymia was seen to mediate suboptimal decision-making. Performance on the IGT showed that alexithymic problem gamblers had deficits in decision-making resulting in a significantly lower overall score (mean $=-19.7, S D=26.7)$, than the non-alexithymic problem gamblers $($ mean $=6.8, S D=12)$. The replicated result makes the mediating effect of alexithymia a convincing interpretation worthy of further research.

In this study, we failed to find evidence of a direct relationship between loss chasing and problem gambling. Dividing participant between low risk and at risk did not lead to a significant difference in the ANOVAs. Nor was there a correlation between the PGSI and the overall losschasing score. It is possible that this failure to find an association between risk of problem gambling and alexithymia can be attributed to the specific characteristics of within-session chasing examined in this study. Palomäkia, Laakasuo, and Salmela (2013) do report a link between loss chasing and problem gambling within task in their examination of poker play. However, they did not examine the level of alexithymia of the participants. Thus, it remains possible that loss-chasing behavior stems from the emotional problems associated with alexithymia and is only coincidental with problem gambling.

One final finding of interest is the correlational analysis that examined which aspects of alexithymia are related to both between- and within-session loss chasing. Overall, both types of loss chasing were associated with alexithymia overall. However, the pattern of correlations for the alexithymia subscales was not the same for between and within loss chasing. For between-session loss chasing, an emotion component was important as was the EOT factor. For the within-session loss chasing, the two emotion components were positively correlated, but the EOT factor was not directly related.

There are a number of important limitations to this study. First, loss chasing was measured on an experimentally controlled task, the CGT. While this allowed us to examine loss chasing in an easily measurable manner, it is not completely clear how this would map on to more realistic situations, such as playing poker online or playing on a fixed odds betting terminal in a casino. Second, alexithymia, as measured by the TAS-20, is a continuous variable representing a normally distributed personality trait, and a fuller analysis of the suggested relationship using more appropriate statistical techniques would require a substantially larger sample. Third, the sample we took was opportunistic and this means that we did not have participants who were known to be receiving treatment for problem gamblers. Nor did we have participants who had self-requested being banned from the bookmakers' shops. Thus, we might be underestimating the importance of loss chasing for the group who is most severely affected by problem gambling. Fourth, given the sample's relatively small size, we were unable to consider several other personality factors, such as sensation seeking, general risk taking, and impulsivity that might impact on loss-chasing behavior.

While neither of the authors of this article are clinicians, we believe that the research reported here may have implications for the treatment of problem gamblers. As many clinicians will be aware, considering whether the problem gambler is high in alexithymia is a useful indicator of possible treatment regimens. Nearly, one third of those at risk of problem gambling were found to be high in alexithymia and those participants who were high in alexithymia were most likely to chase losses. Thus, one aspect of problem gambling behavior, loss chasing, may reflect a symptom of an underlying inability to effectively process emotions. However, people who are high in alexithymia are notoriously difficult to treat using emotion-focused treatments (Ogrodniczuk, Piper, \& Joyce, 2011; Taylor \& Bagby, 2013). Given this, then any treatment of problem gamblers who are high in alexithymia may benefit from focusing either directly on the loss-chasing behavior or on helping such people to make sense of their emotional reactions. In the former case, cognitive behavioral therapy may be best suited to the task. It has already been shown to be a relatively successful treatment (Tolchard, 2017) and focusing attention on the loss-chasing behavior may well benefit problem gamblers who happen to be high in alexithymia. With respect to the latter, Lane, Weihs, Herring, Hishaw, and Smith (2015) suggest that alexithymia reflects a problem in linking the emotional stimulus to a mental representation of the ensuing emotional state. Thus, a strategy that emphasizes the emotional consequences of loss and helps build a mental representation of the negative consequences of that loss might be successful.

In summary, notwithstanding the above limitations, the research presented here confirms a relationship between alexithymia and risk of problem gambling. However, it moves this research one step closer to understanding a specific aspect of problem gambling, loss chasing. Following suggestions by Bibby and Ferguson (2011) and Bibby (2016), we argue that loss-chasing behavior is more strongly related to alexithymia than problem gambling in general and this arises because people who are high in alexithymia are less sensitive to losses; they are less loss averse. Specifically, we suggest it is the failure to respond appropriately to the emotional consequence of losses that lead some problem gamblers to loss chase both between- and within-gambling sessions.

Funding sources: No financial support was received for this study. 
Authors' contribution: The lead author was primarily responsible for the study concept and design, analysis and interpretation of data, and statistical analysis. The second author was primarily responsible for the data collection. Both authors made substantial contributions to the writing and development of the final submitted paper. Both authors also had full access to all data in the study and take responsibility for the integrity of the data and the accuracy of the data analysis.

Conflict of interest: The authors declare no conflict of interest.

\section{REFERENCES}

Aïte, A., Barrault, S., Cassotti, M., Borst, G., Bonnaire, C., Houde, O., Varesco, I., \& Moutier, S. (2014). The impact of alexithymia on pathological gamblers' decision making: A preliminary study of gamblers recruited in "Sportsbook" Casinos. Cognitive Behavioral Neurology, 27(2), 59-67. doi:10.1097/ WNN.0000000000000027

American Psychiatric Association. (2013). Diagnostic and statistical manual of mental disorders (5th ed.). Washington, DC: American Psychiatric Association.

Bagby, M., \& Taylor, G. (1997). Measurement and validation of the alexithymia construct. In G. Taylor, R. Bagby, J. Parker, \& J. Grotstein (Eds.), Disorders of affect regulation: Alexithymia in medical and psychiatric illness (pp. 46-66). Cambridge, UK: Cambridge University Press.

Bagby, R. M., Parker, J. D. A., \& Taylor, G. J. (1994). The 20 -item Toronto-Alexithymia-Scale. 1. Item selection and cross-validation of the factor structure. Journal of Psychosomatic Research, 38(1), 23-32. doi:10.1016/0022-3999 (94)90005-1

Bechara, A., Damasio, A. R., Damasio, H., \& Anderson, S. W. (1994). Insensitivity to future consequence following damage to human pre-frontal cortex. Cognition, 50(1-3), 7-15. doi:10.1016/0010-0277(94)90018-3

Bibby, P. A. (2016). Loss-chasing, alexithymia and impulsivity: Alexithymia as a precursor to problem gambling. Frontiers in Psychology, 7, 3. doi:10.3389/fpsyg.2016.00003

Bibby, P. A., \& Ferguson, E. (2011). The ability to process emotional information predicts loss aversion. Personality and Individual Differences, 51(3), 263-266. doi:10.1016/j. paid.2010.05.001

Bonnaire, C., Barrault, S., Aïte, A., Cassotti, M., Moutier, S., \& Varescon, I. (2017). Relationship between pathological gambling, alexithymia and gambling type. The American Journal on Addictions, 26(2), 152-160. doi:10.1111/ ajad. 12506

Bonnaire, C., Bungener, C., \& Varescon, I. (2009). Subtypes of French pathological gamblers: Comparison of sensation seeking, alexithymia and depression scores. Journal of Gambling Studies, 25(4), 455-471. doi:10.1007/s10899-009-9142-z

Bonnaire, C., Bungener, C., \& Varescon, I. (2013). Alexithymia and gambling: A risk factor for all gamblers? Journal of Gambling Studies, 29(1), 83-96. doi:10.1007/s10899-012-9297-x

Breen, R. B., \& Zuckerman, M. (1999). 'Chasing' in gambling behavior: Personality and cognitive determinants. Personality and Individual Differences, 27(6), 1097-1111. doi:10.1016/ S0191-8869(99)00052-5

Di Trani, M., Renzi, A., Vari, C., Zavattini, G. C., \& Solano, L. (2017). Gambling disorder and affect regulation - The role of alexithymia and attachment style. Journal of Gambling Studies, 33(2), 649-659. doi:10.1007/s10899-016-9637-3

Dickerson, M., Hinchy, J., \& Fabre, J. (1987). Chasing, arousal and sensation seeking in off-course gamblers. British Journal of Addiction, 82(6), 673-680. doi:10.1111/j.1360-0443.1987. tb01530.x

Ferguson, E., Bibby, P. A., Rosamond, S., O'Grady, C., Parcell, A., Amos, C., \& O'Carroll, R. (2009). Alexithymia, cumulative feedback, and differential response patterns on the Iowa gambling task. Journal of Personality, 77(3), 883-902. doi:10.1111/j.1467-6494.2009.00568.x

Ferris, J., \& Wynne, R. (2001). The Canadian Problem Gambling Index: Final report. Ottawa, ON: The Canadian Consortium for Gambling Research.

Lane, R. D, Weihs, K. L., Herring, A., Hishaw, A., \& Smith, R. (2015). Affective agnosia: Expansion of the alexithymia construct and a new opportunity to integrate and extend Freud's legacy. Neuroscience and Biobehavioral Reviews, 55, 594-611. doi:10.1016/j.neubiorev.2015.06.007

Lesieur, H. R., \& Blume, S. B. (1987). The South Oak Gambling Screen (SOGS) - A new instrument for the identification of pathological gamblers. The American Journal of Psychiatry, 144(9), 1184-1188. doi:10.1176/ajp.144.9.1184

Linnet, J., Rojskjaer, S., Nygaard, J., \& Maher, B. A. (2006). Episodic chasing in pathological gamblers using the Iowa gambling task. Scandinavian Journal of Psychology, 47(1), 43-49. doi:10.1111/j.1467-9450.2006.00491.x

Lumley, M. A., \& Roby, K. J. (1995). Alexithymia and pathological gambling. Psychotherapy and Psychosomatics, 63(3-4), 201-206. doi:10.1159/000288960

Maniaci, G., Picone, F., Dimarco, T, Lipari, A., Brancato, A., \& Cannizaro, C. (2015). Psychodiagnostic assessment of pathological gamblers: A focus on personality disorders, clinical syndromes and alexithymia. International Journal of Mental Health and Addiction, 13(6), 728-739. doi:10.1007/s11469015-9550-5

Mitrovic, D. V., \& Brown, J. (2009). Poker mania and problem gambling: A study of distorted cognitions, motivation and alexithymia. Journal of Gambling Studies, 25(4), 489-502. doi:10.1007/s10899-009-9140-1

O'Connor, J., \& Dickerson, M. (2003). Impaired control over gambling in gaming machine and off-course gamblers. Addiction, 98(1), 53-60. doi:10.1046/j.1360-0443.2003.00232.x

Ogrodniczuk, J. S., Piper, W. E., \& Joyce, A. S. (2011). Effect of alexithymia on the process and outcome of psychotherapy: A programmatic review. Psychiatry Research, 190(1), 43-48. doi:10.1016/j.psychres.2010.04.026

Palomäkia, J., Laakasuo, M., \& Salmela, M. (2013). 'This is just so unfair!': A qualitative analysis of loss-induced emotions and tilting in on-line poker. International Gambling Studies, 13(2), 255-270. doi:10.1080/14459795.2013.780631

Parker, J. D. A., Keefer, K. V., Taylor, G. J., \& Bagby, R. M. (2008). Latent structure of the alexithymia construct: A taxometric investigation. Psychological Assessment, 20(4), 385-396. doi:10.1037/a0014262

Parker, J. D. A., Taylor, G. J., \& Bagby, R. M. (2001). The relationship between emotional intelligence and alexithymia. 
Personality and Individual Differences, 30(1), 107-115. doi:10.1016/S0191-8869(00)00014-3

Parker, J. D. A., Wood, L. M., Bond, B. J., \& Shaughnessy, P. (2005). Alexithymia in young adulthood: A risk factor for pathological gambling. Psychotherapy and Psychosomatics, 74(1), 51-55. doi:10.1159/000082027

Rogers, R. D., Everitt, B. J., Baldacchino, A., Blackshaw, A. J., Swainson, R., Wynne, K., Baker, N. B., Hunter, J., Carthy, T., Booker, E., London, M., Deakin, J. F., Sahakian, B. J., \& Robbins, T. W. (1999). Dissociable deficits in the decisionmaking cognition of chronic amphetamine abusers, opiate abusers, patients with focal damage to prefrontal cortex, and tryptophan-depleted normal volunteers: Evidence for monoaminergic mechanisms. Neuropsychopharmacology, 20(4), 322-339. doi:10.1016/S0893-133X(98)00091-8

Sifneos, P. E. (1973). The prevalence of 'alexithymic' characteristics in psychosomatic patients. Psychotherapy and
Psychosomatics, 22(2-6), 255-262. doi:10.1159/0002 86529

Taylor, G. J., \& Bagby, R. M. (2013). Psychoanalysis and empirical research: The example of alexithymia. Journal of the American Psychoanalytic Association, 61(1), 99-133. doi: $10.1177 / 0003065112474066$

Taylor, G. J., Bagby, R. M., \& Parker, J. D. A. (1997). Disorders of affect regulation: Alexithymia in medical and psychiatric illness. Cambridge, UK: Cambridge University Press.

Tolchard, B. (2017). Cognitive-behavior therapy for problem gambling: A critique of current treatments and proposed new unified approach. Journal of Mental Health, 26(3), 283-290. doi:10.1080/09638237.2016.1207235

Toneatto, T., Lecce, J., \& Bagby, M. (2009). Alexithymia and pathological gambling. Journal of Addictive Diseases, 28(3), 193-198. doi:10.1080/10550880903014775 